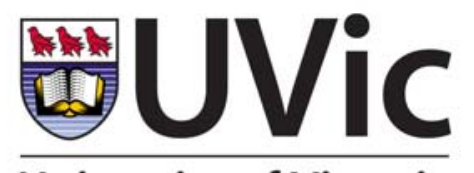

University of Victoria

Econometrics Working Paper EWP1202

ISSN 1485-6441

Department of Economics

\title{
Constructing Confidence Bands for the Hodrick-Prescott Filter
}

\author{
David E. Giles \\ Department of Economics, University of Victoria
}

April 2012

\begin{abstract}
By noting that the Hodrick-Prescott filter can be expressed as the solution to a particular regression problem, we are able to show how to construct confidence bands for the filtered time-series. This procedure requires that the data are stationary. The construction of such confidence bands is illustrated using annual U.S. data for real value-added output; and monthly U.S. data for the unemployment rate.
\end{abstract}

Keywords: $\quad$ Hodrick-Prescott filter; time-series decomposition; confidence bands

JEL classifications: $\quad$ C13; C20; E3

\section{Author Contact:}

David E. Giles, Dept. of Economics, University of Victoria, P.O. Box 1700, STN CSC, Victoria, B.C., Canada V8W 2Y2; e-mail: dgiles@uvic.ca; Phone: (250) 721-8540; FAX: (250) 721-6214 


\section{Introduction}

The Hodrick-Prescott (H-P) filter is very widely used for the decomposition of economic timeseries into their trend and cyclical components. Although economists generally attribute this filter to Hodrick and Prescott $(1980,1997)$, it actually dates at least from Leser (1961), and it is based on much earlier contributions by Whittaker (1922) and by Henderson (1924).

Although the weaknesses of the H-P filter are well-documented, and various competing filters are available, it remains one of the standard tools used by empirical macroeconomists. The application of the H-P filter to extract the trend from a time-series amounts to signal extraction. Similarly, the estimation of a regression model extracts a signal about the dependent variable from the data, and separates it from the "noise". In the case of a regression model it would be unthinkable to report estimated coefficients without their standard errors; or predictions without confidence bands. So, it is somewhat surprising that the trend that we extract from a time-series using the H-P filter is always reported without any indication of the uncertainty associated with it.

In this paper we show how asymptotically valid confidence bands can be constructed for the H-P filter. The key insight is to recognize that the H-P filter can be represented as the solution to a regression problem. This interpretation of the filter is discussed in the next section. Section 3 illustrates the application of our results using U.S. unemployment rate data. Our conclusions appear in section 4 .

\section{A regression interpretation}

Suppose that we have a stationary time-series, $y_{t}$, for $t=1,2,3, \ldots ., T$. We assume that the data can be described as $y_{t}=\tau_{t}+c_{t}$, where $\tau_{t}$ represents the non-linear trend in the series, and $c_{t}$ is the cyclical component. A multiplicative representation of the time-series can be accommodated by taking the logarithms of the data.

Then, the H-P filter involves solving the following optimization problem:

$$
\min _{\left(\tau_{t}\right)}\left\{\left\{\sum_{t=1}^{T}\left(y_{t}-\tau_{t}\right)^{2}+\lambda \sum_{t=2}^{T-1}\left[\left(\tau_{t+1}-\tau_{t}\right)^{2}+\left(\tau_{t}-\tau_{t-1}\right)^{2}\right]\right\},\right.
$$

for $t=1$ to $T$. 
The first term in the objective function in (1) can be viewed as measuring "goodness of fit"; while the second term imposes a penalty for "roughness". The smoothing parameter, $\lambda$, is chosen by the user and there are well-known rules regarding its choice, depending on the frequency of the data. Beginning with Danthine and Girardin (1989), several authors have noted that the optimization problem (1) can be re-written in the following vector-matrix form:

$$
\min _{(\tau)} .\left\{\left(c^{\prime} c+\lambda(K \tau)^{\prime}(K \tau)\right\} .\right.
$$

Here, $c$ and $\tau$ are $(T \times 1)$ vectors with typical elements $c_{t}$ and $\tau_{t}$ respectively; and $K=\left\{k_{i j}\right\}$ is a $[(T-2) \times T]$ "second-differencing” matrix, with

$$
\begin{aligned}
k_{i j} & =1 \text { (if } i=j, \text { or } j=i+2) \\
& =-2(\text { if } j=i+1) \\
& =0 \text { (otherwise). }
\end{aligned}
$$

The solution to the problem in (2) is:

$$
\hat{\tau}=\left[I_{T}+\lambda K^{\prime} K\right]^{-1} y,
$$

where $I_{T}$ is an identity matrix of order $T$. (In practice, care has to be taken over the inversion of the matrix in (3), as it can be close to being singular.)

We see from (3) that the H-P filter can be interpreted as an application of Ridge Regression. Specifically, if we consider the "regression model"

$$
y=I_{T} \tau+c,
$$

then any (generalized) ridge estimator of $\tau$ is of the general form:

$$
\tilde{\tau}=\left[I_{T}^{\prime} I_{T}+\lambda A\right]^{-1} I_{T}^{\prime} y=\left[I_{T}+\lambda A\right]^{-1} y,
$$

for some positive semi-definite matrix, $A$. Setting $A=K^{\prime} K$ in (5), we see that $\tilde{\tau}=\hat{\tau}$.

Schlicht (2005) extended this analysis to allow for the simultaneous estimation of the smoothing parameter, $\lambda$, and $\left\{\tau_{t}\right\}_{t=1}^{T}$. However, this possibility is not pursued here. 
It is also clear that, when written in the form (3), the H-P filter can also has a Bayesian interpretation, as was noted originally by Ley (2006), and more recently by Polasek (2011). If the cyclical component in (4) is assumed to be normally distributed with a variance of $\sigma^{2}$ and we use the natural-conjugate prior for the "parameters", so that $p(\tau \mid \sigma) \sim \mathrm{N}\left[0,\left(\sigma^{2} / \lambda\right)\left(K^{\prime} K\right)^{-1}\right]$, and $p(\sigma)$ is inverted-gamma, then the Bayes estimator of $\boldsymbol{\tau}$ is given by (3).

As the H-P filter can be interpreted as an estimator for a particular regression model, we can easily construct the covariance matrix for this estimator. From this, we can get confidence intervals for each value in the $\boldsymbol{\tau}$ series. That is, we can obtain a confidence band for the extracted trend component.

From (3), note that the covariance matrix for the elements of $\hat{\tau}$ is given by

$$
V(\hat{\tau})=\left[I_{T}+\lambda K^{\prime} K\right]^{-1} V(y)\left[I_{T}+\lambda K^{\prime} K\right]^{-1} .
$$

The form of $V(y)$ will depend on the particular time-series being filtered, and under suitable assumptions this covariance matrix can be estimated from the data. The square roots of the diagonal elements of the estimated matrix corresponding to (6) will be asymptotic standard errors and a 95\% (say) confidence band series for the extracted trend can be constructed as $\left\{\hat{\tau}_{t}-1.96\right.$ s.e. $\left(\hat{\tau}_{t}\right), \hat{\tau}_{t}+1.96$ s.e. $\left.\left(\hat{\tau}_{t}\right)\right\} ; t=1,2, \ldots, T$.

In general, it would be unrealistic to assume that $V(y)=\sigma^{2} I$. Instead, if the data are stationary, then an ARIMA model for the series can be identified and estimated, yielding an estimate of the $V(y)$ matrix for substitution into (6). The stationarity of the data is crucial requirement to the application of (6). This imposes an important limitation on this analysis. For example, real GDP for most countries is I(1), so in that context confidence bands for the H-P filter could be constructed for output growth, but not for output itself.

\section{Applications}

We consider three applications of these results. In each case, application of the (augmented) Dickey-Fuller and KPSS tests indicates that the data are stationary. The associated EViews workfiles and program files can be downloaded from web.uvic.ca/ dgiles/downloads/hp_filter/. Our first example relates to multifactor productivity. Specifically, we consider the annual rate of 
growth in real value-added output (private business sector, excluding government enterprises) for the U.S. over the period 1949 to 2010. See Bureau of Labor Statistics (2012).

The data and the H-P filtered trend, obtained using the EViews package and $\lambda=100$, are shown in Figure 1. The correlogram for the series indicates that it is white noise, so Figure 2 provides $95 \%$ confidence bands constructed using equation (6) with $V(y)=\sigma^{2} I$, and with $\sigma^{2}$ estimated by the sample variance.

Our second application relates to the seasonally adjusted unemployment rate for all full-time U.S. workers. The decomposition of such data is of some interest as it offers one way of measuring the NAIRU. We use the monthly time-series, LNS14100000, from the FRED database (Federal Reserve Bank of St. Louis, 2012), for the period 1968M01 to 2012M03.

Using EViews to apply the H-P filter with the value $(14,400)$ of $\lambda$ chosen according to the Ravn and Uhlig (2002) criterion for monthly data, we obtain the results in Figure 3. Under the very restrictive assumption that $V(y)=\sigma^{2} I$, estimating $\sigma^{2}$ by using the sample variance of the original data, equation (6) yields the 95\% confidence bands shown in Figure 4.

However, the correlogram for the unemployment rate $(U)$ data suggests that this series can be modeled by an AR(4) process. Simplifying the model using the SIC, the following restricted AR(4) process was selected:

$$
\hat{U}_{t}=0.0779+1.1834 U_{t-1}-0.1953 U_{t-4} \quad ; \quad \bar{R}^{2}=0.9908 \quad ; \quad s^{2}=0.03123
$$

Asymptotic standard errors appear in parentheses, and the roots of the characteristic equation for this autoregression lie outside the unit circle. To construct $V(y)$ in this case, we use the results of Hamilton (1994, pp.58-59), and his exercise 10.1 (p.290). His $F$ matrix (p.7) is constructed using $\phi_{1}=1.1834, \phi_{2}=\phi_{3}=0$, and $\phi_{4}=-0.1953$, and $s^{2}$ in the above regression results provides a consistent estimator of $\sigma^{2}$. Then, using equation (6) we obtain the (much wider) 95\% confidence bands shown in Figure 5. The importance of reporting the confidence bands can be seen by considering the H-P trend value of 9.34\% in February 1983, when the 95\% confidence interval was $(6.24 \%, 12.44 \%)$ 
Fig. 1 U.S. Value-Added Output Growth: Private Business Sector (annual \% change)

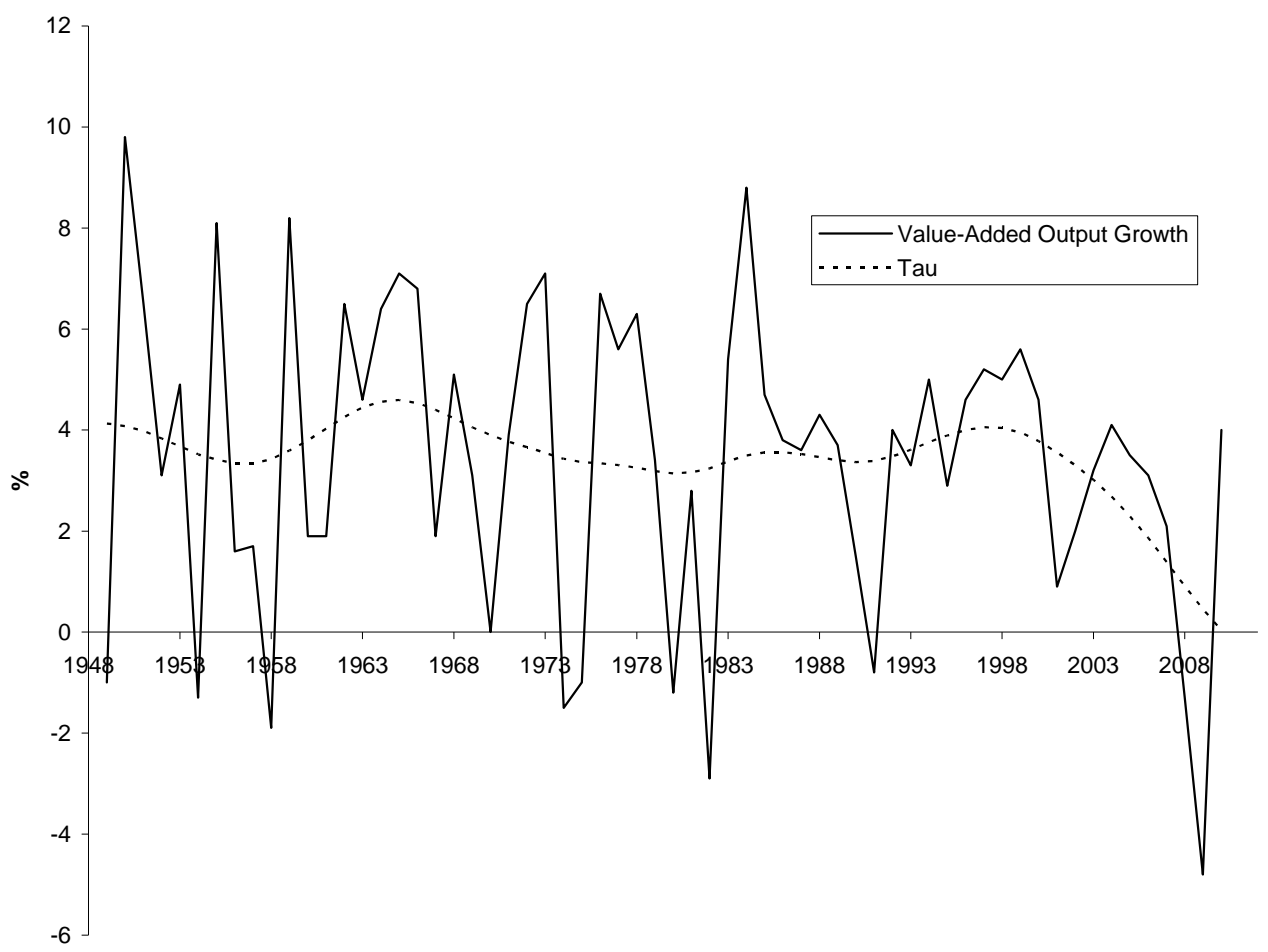

Fig. 2 U.S. Value-Added Output Growth: H-P Filtered Trend and $95 \%$ Confidence Band

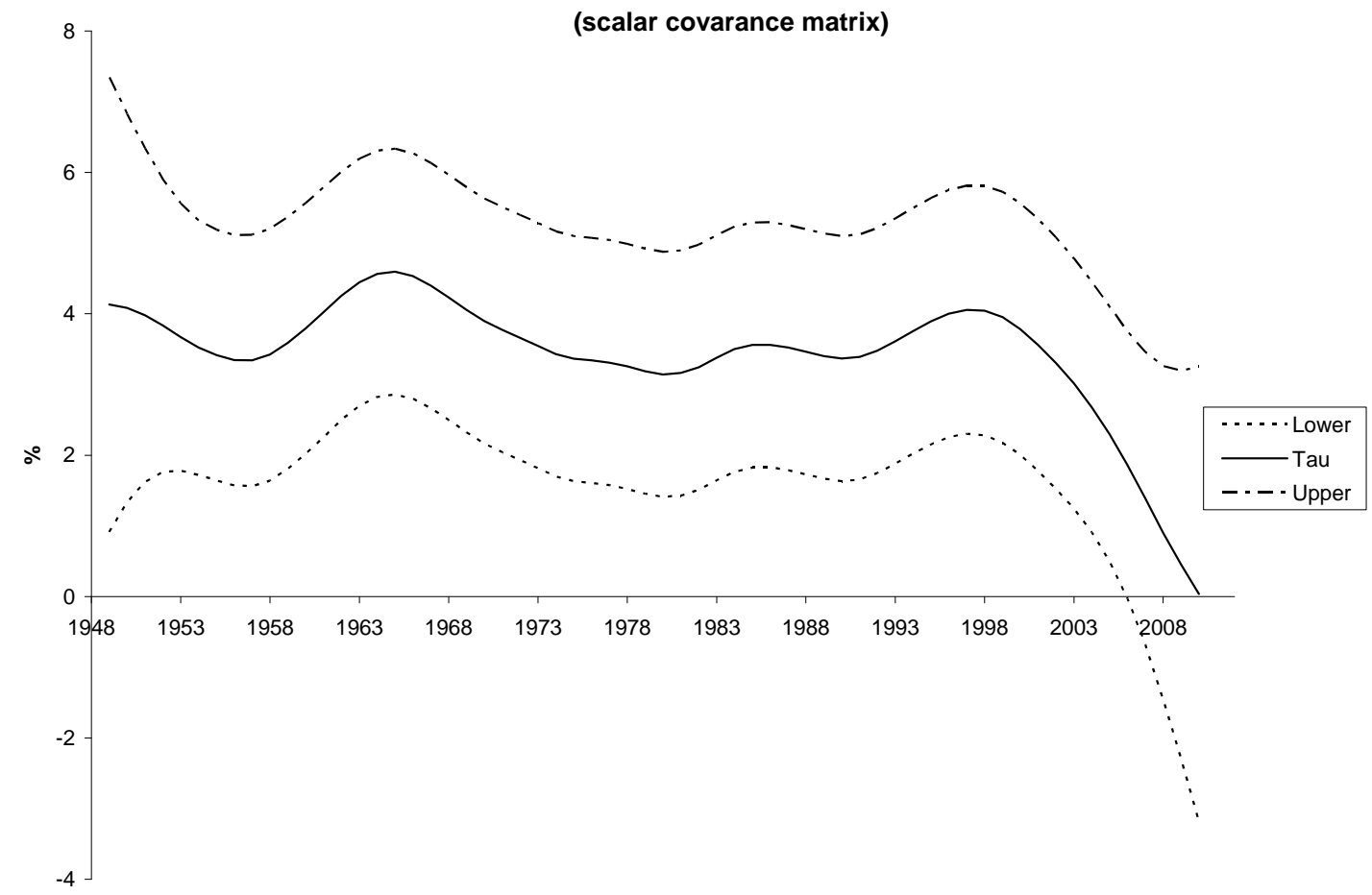


Fig. 3 U.S. Unemployment Rate: Full-Time Workers

(Seasonally Adjusted)

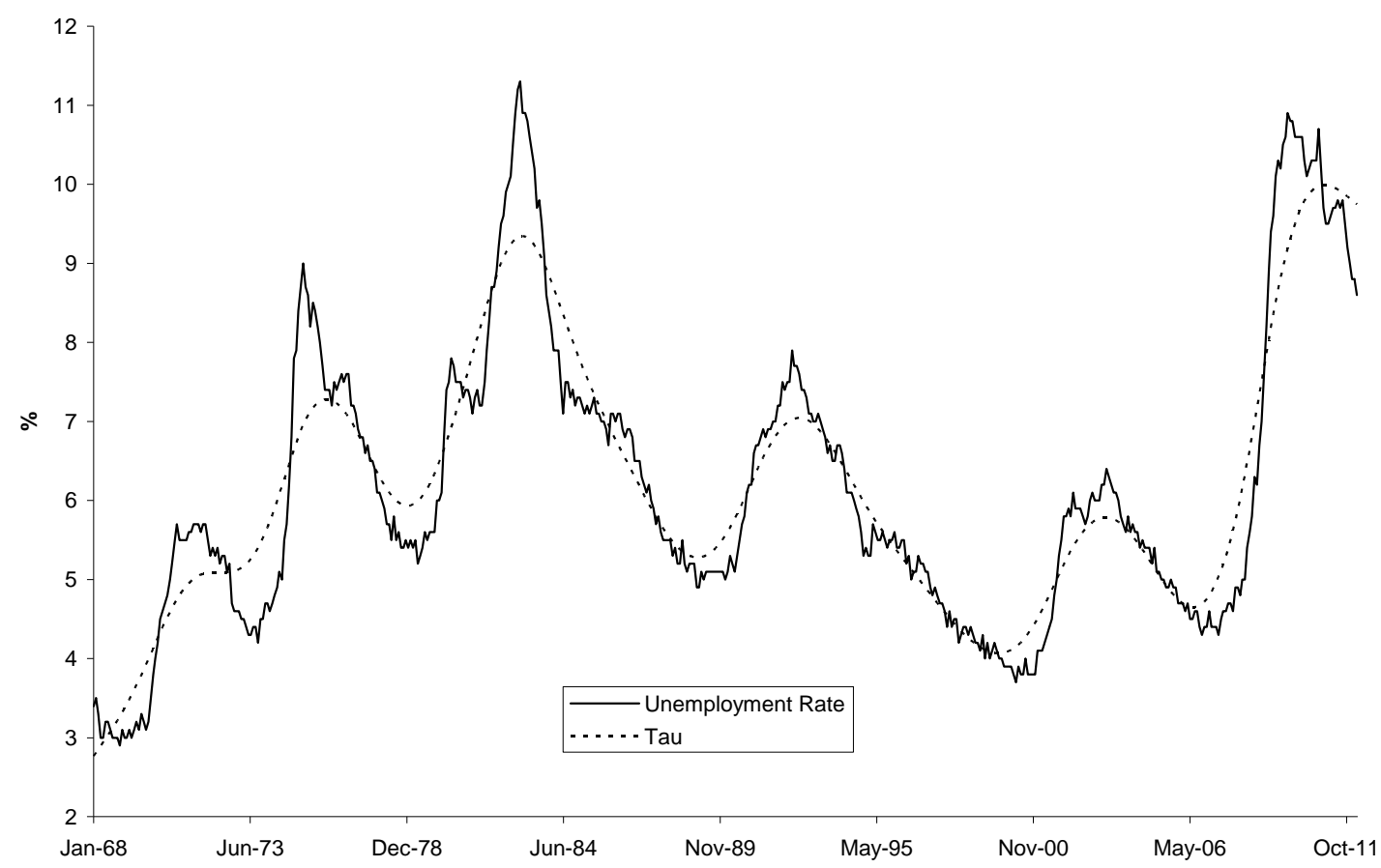

Fig. 4 U.S. Unemployment Rate: H-P Filtered Trend and $95 \%$ Confidence Band

(scalar covariance matrix)

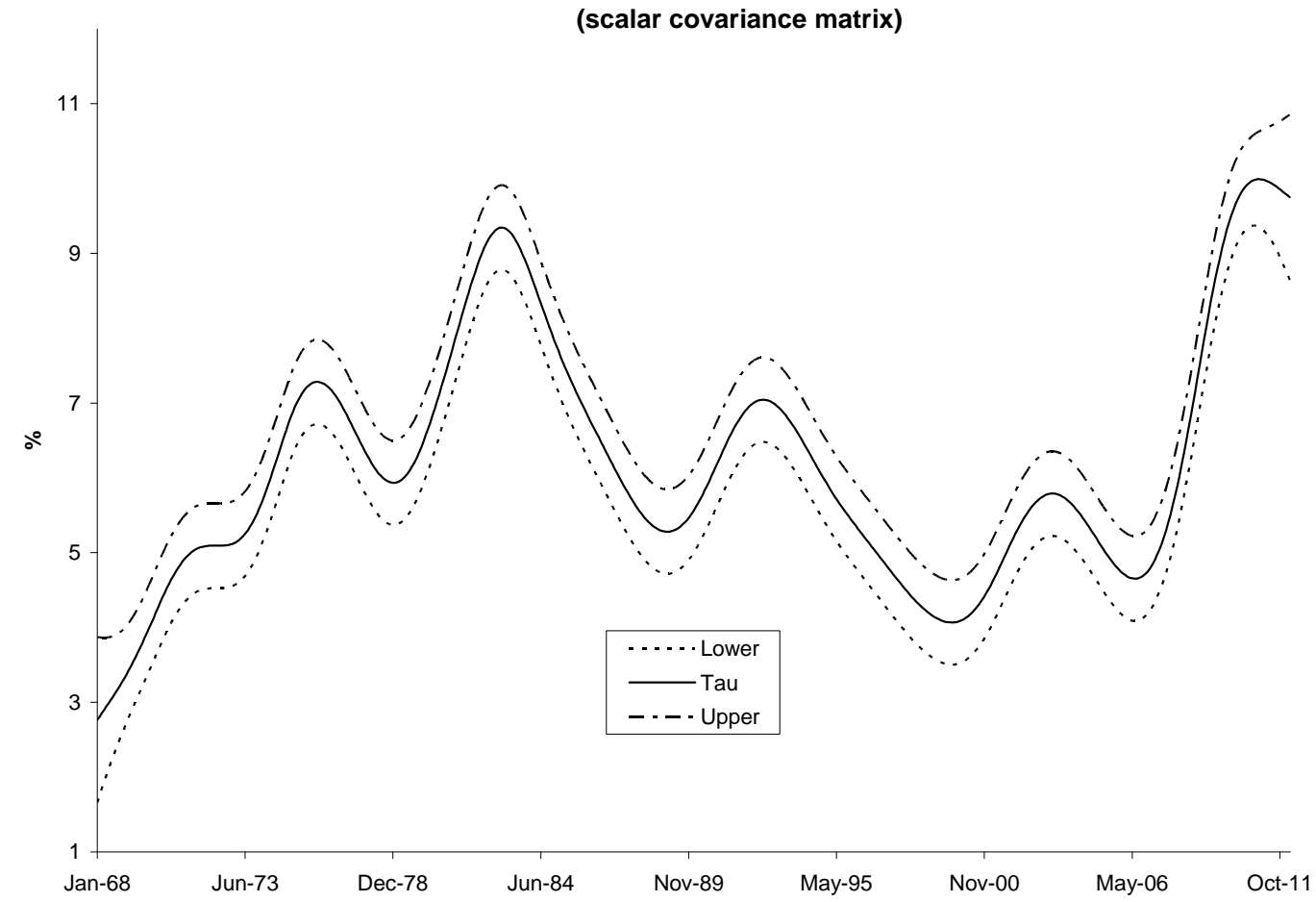


Fig. 5 U.S. Unemployment Rate: H-P Filtered Trend and $95 \%$ Confidence Band (restricted AR(4) model)

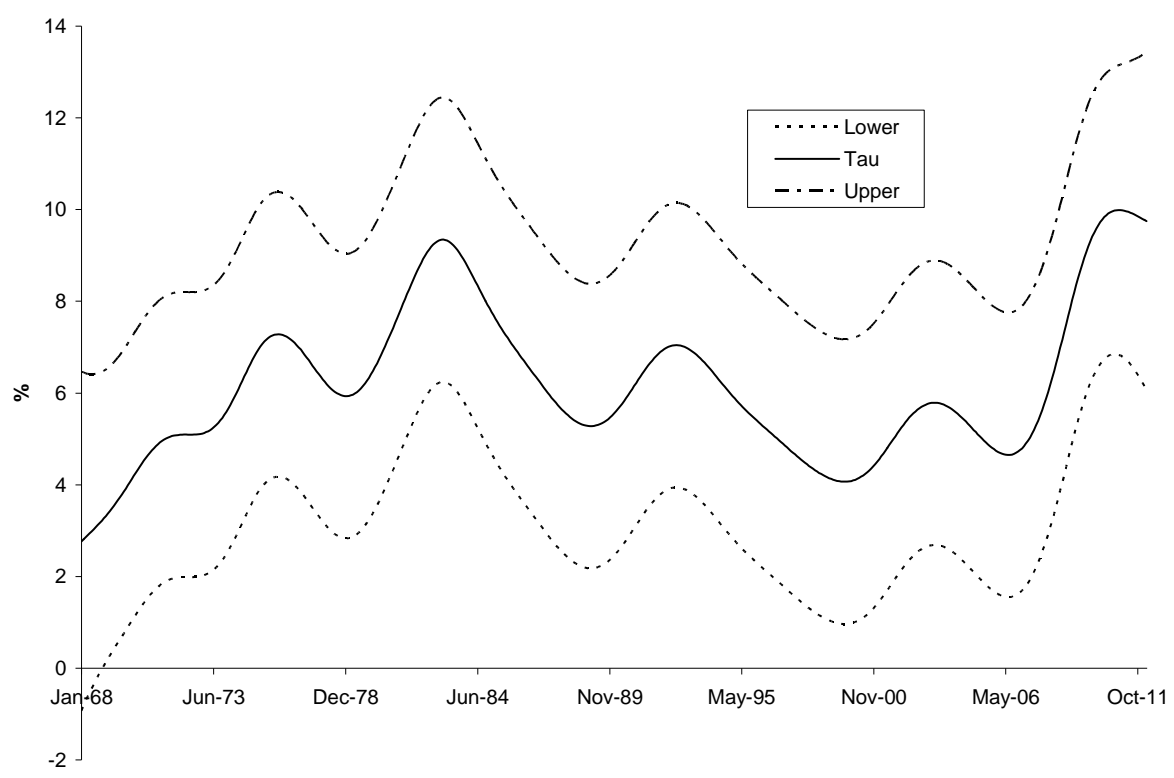

\section{Conclusions}

We have shown that asymptotically valid confidence bands can be constructed very easily for trend series extracted using the Hodrick-Prescott filter. This is achieved by noting that this filter can be viewed as the solution to a ridge regression problem. We have illustrated the application of these results to macroeconomic data of types that are commonly subjected to the HodrickPrescott filter.

\section{Acknowledgement}

I am grateful to Riccardo (Jack) Luccetti and Graham Voss for their helpful comments on an informal earlier version of this work. 


\section{References}

Bureau of Labor Statistics, 2012. Multifactor productivity. http://www.bls.gov/mfp/. Accessed 16 April 2012.

Danthine, J-P., Girardin, M., 1989. Business cycles in Switzerland. European Economic Review 33, 31-50.

Federal Reserve Bank of St. Louis, 2012. FRED data. http://research.stlouisfed.org/fred2/. Accessed 13 April 2012.

Hamilton, J.D., 1994. Time Series Analysis. Princeton University Press, Princeton, NJ.

Henderson, R., 1924. A new method of graduation. Transactions of the Actuarial Society of America 25, 29-40.

Hodrick, R.J., Prescott, E.C. 1980. Postwar U.S. business cycles: An empirical investigation. Discussion Paper No. 451, Department of Economics, Carnegie Mellon University.

Hodrick, R.J., Prescott, E.C., 1997. Postwar U.S. business cycles: An empirical investigation. Journal of Money, Credit, and Banking 29, 1-16.

Leser, C.E.V., 1961. A simple method of trend construction. Journal of the Royal Statistical Society, B 23, 91-107.

Ley, E., 2006. The Hodrick-Prescott filter. Knowledge Brief for Bank Staff. The World Bank, Washington DC.

Polasek, W., 2011. The Hodrick-Prescott (HP) filter as a Bayesian regression model. WP 11-46, The Rimini Centre for Economic Analysis, Rimini, Italy.

Ravn, M.O., Uhlig, H., 2002. On adjusting the Hodrick-Prescott filter for the frequency of observations. Review of Economics and Statistics 84, 371-376.

Schlicht, E., 2005. Estimating the smoothing parameter in the so-called Hodrick-Prescott filter. Journal of the Japan Statistical Society 35, 99-119.

Whittaker, E.T., 1922. On a new method of graduation. Proceedings of the Edinburgh Mathematical Society 41, 63-75. 